\title{
A Novel Two stage Cascading Algorithm for Fingerprint Recognition
}

\author{
Xuying Zhao, Xiaokun Zhang, Huamin Feng, Geng Zhao, Kejun Zhang and Rong Qian \\ Department of Computer Science and Technology \\ Beijing Electronic Science and Technology Institute \\ NO.7 Fufeng Road, Fengtai District, Beijing, P.R.China \\ \{xyzhao, sam, fenghm, zg, zkj, rqian\}@ besti.edu.cn
}

\begin{abstract}
A novel two stage cascading algorithm for fingerprint recognition is proposed combining minutiae based classifier with orientation based classifier. Fingerprint images are classified at the first stage by using the minutiae based method presented in this paper. At the second stage, there is a small probability in application that both the results of minutiae and orientation based matchers are utilized as inputs of the higher stage classification system. Experiments have been conducted on public database of fingerprint images and the results reveal that the approach described in this paper can achieve good performance with higher accuracy and better efficiency.
\end{abstract}

Keywords-fingerprint recognition; cascading; minutiae; orientation; information fusion

\section{INTRODUCTION}

Although high matching accuracy can be achieved on databases with good quality fingerprint images in the fingerprint recognition systems nowadays, there are still improvements required for accurately matching fingerprint images of low quality. Furthermore, the design of cooperative and computationally efficient matching algorithms is still a challenging problem [1].

In general, a full fingerprint recognition system involves feature extraction and matching which are two critical components having an important effect on the performance of the system. According to the features that is used to represent fingerprints, fingerprint classifier can be divided into optical correlation based, transform feature based, graph or structural information based, orientation based, minutiae based, and level 3 features, i.e. pores and ridge contours based [2]. Algorithms based on Minutiae matching do not perform well sometimes that relate to ridge activity in the locality of minutiae points because of the inadequate number of corresponding points in the input and stored reference images [3]. Therefore, complementary information is provided for improving the accuracy, while most of the fingerprint recognition systems depend on minutiae matching.

A multistage method of information fusion, namely cascading, is discussed in $[4,5]$ that a sequence of classifiers are ordered under some conditions where the next classifiers is only considered for patterns refused by the previous classifiers. The idea of building a multi-stage pattern classification system with refection can give less decision risk than a single-stage classifier for that the reject patterns can be dealt with at another higher-stage pattern recognition system that would utilize more informative measurements in which new features are derived [4]. Moreover, a cascading system is developed where an earlier classifier handles major cases and a complex classifier is only utilized with a small possibility hence not increasing the complexity greatly [5]. Therefore, a cascading scheme for iris recognition is presented by Sun [6] and the experimental results of decision-level classifiers combination demonstrate that the cascading classification system outperforms single classifier. Additionally, a registration method cascading two fingerprint registration schemes is designed by Qi [7] and a series of experiments validate the effectiveness of the multi-stage strategy.

Inspired by the idea of constructing multi-stage cascading of multiple classifiers, a two stage cascading scheme is proposed to combine minutiae based fingerprint matching with orientation based method. Fingerprint matching based on minutiae can be regarded as searching for identical triangle frameworks with minutiae at the vertices in input and template images [8]. The image is divided into a series of circulars by using the belief function of fingerprint singularity to decrease computational and space complexity of finding corresponding triangles. Accordingly, minutiae based method of triangular matching integrated with singular features is presented in this paper. In addition, orientation fields that are complementary to minutiae information can be estimated from fingerprint images that snap the global appearance of fingerprints [9]. Therefore, information fusion is achieved at the confidence level by normalization of orientation matching scores integrated with minutiae matching scores.

The rest of this paper is organized as follows. In section2, we discuss minutiae based triangle matching with belief function. Orientation based method of matching and normalization is described in section 3 . Section 4 presents our two stage matching algorithm for fingerprint recognition. In section 5, experimental results and conclusions are reported.

\section{MinUtiAE BASED MATCHING}

In mathematics, fingerprint matching based on minutiae is essentially to find the isomorphic subsets as many minutiae as possible in input and template fingerprint image. The minutiae isomorphic subsets of fingerprint are in possession of the same overall geometric configuration. 
Therefore, the process of fingerprint matching is identical to search for a largest geometric configuration. To describe the properties of geometric configuration, triangular framework is defined as an exceptional polygon framework that is composed of only triangles with at least a public edge in neighborhood, such as the framework ABCDEF, as is shown in Fig. 1.

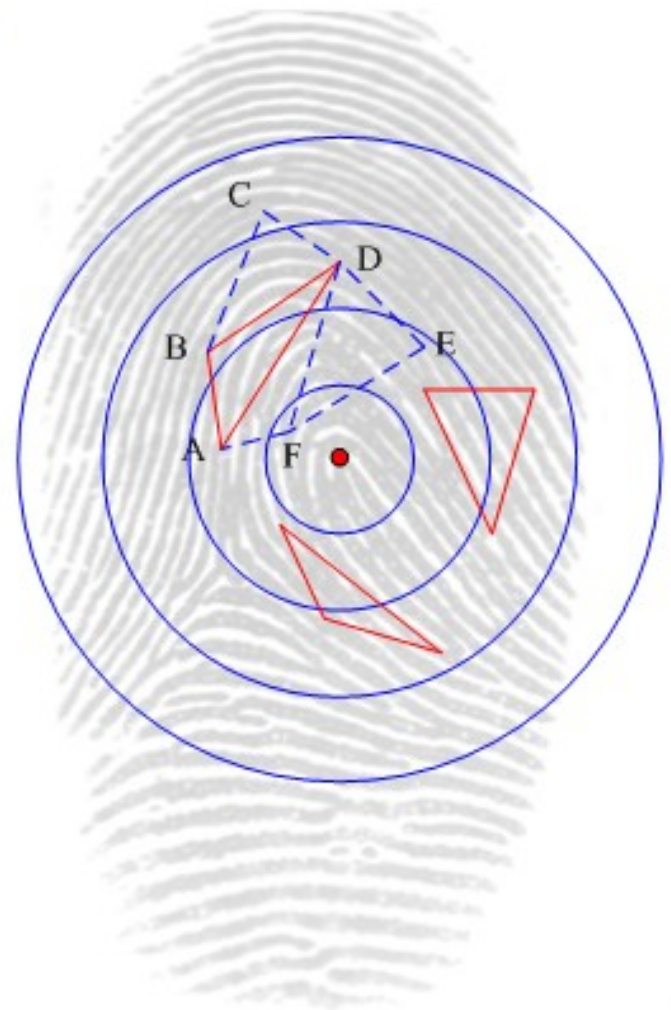

Figure 1. Triangle frameworks and seed triangles in separataed area.

It can be verified that identical triangular frameworks possess the same overall geometric configuration of their vertices, while two planar graphs that have the same geometric configuration can bring into equivalent triangular frameworks.

Minutiae based fingerprint matching is equivalent to the generation of identical triangular frameworks in which all the minutiae are landed at their vertices. In the process of matching, triangle frameworks are derived and a pair of the triangular framework is chosen as the final matching result that involves the maximum number of minutiae at their vertices. The matching score (Ms) of two fingerprint images can be calculated as

$$
M s=\frac{P^{2}}{M \times N},
$$

in which $\mathrm{P}$ is the number of minutiae at their vertices in the matching triangular framework and $M, N$ is the number of minutiae respectively in two fingerprint images.
To suppress computational cost of identifying correspondent triangles, fingerprint image can be divided into a series of circular areas centered at the singular point by defining the belief function as follows.

$$
\operatorname{Bel}(\mu)= \begin{cases}0.01, & \text { if }|\mu| \leq \mathrm{T}_{\mathrm{s}} \\ \frac{|\mu|}{T_{\mathrm{d}}}, & \text { if } \mathrm{T}_{\mathrm{s}}<|\mu|<\mathrm{T}_{\mathrm{d}}, \\ 1 & \text { if }|\mu| \geq \mathrm{T}_{\mathrm{d}}\end{cases}
$$

where $\mu$ is a certainty measure of singular points [8] as is shown in Fig. 1, while $T_{d}$ is determined experientially. The image is separated into a series of circular areas that are centered at the singular point. The nth radius $r_{n}$ of the corresponding concentric circle is calculated by

$$
\mathrm{r}_{\mathrm{n}}=\mathrm{n} \frac{\mathrm{r}_{0}}{\mathrm{c}(\mu)}
$$

in which $r_{0}$ is limited by the area of sensor capturing the fingerprint. There is no necessary for dividing the image in case $\operatorname{Bel}(\mu)=0.01$.

Only those corresponding triangles pairs are studied whose vertices don't cut across over any circular area and a vertex lands in different circular region while searching for seed triangles. Thereafter, identical triangles are found in the circular area in neighborhood of input fingerprint image and template image. Consequently, the candidate triangle number is greatly reduced while the final triangular frameworks that have the maximum number of vertices will not be affected.

\section{ORIENTATION MATCHING AND NORMALIZATION}

The method of estimating fingerprint orientation fields that describe fingerprint texture information is recommended in [9]. Two fingerprint images are registered by conducting a rigid transformation utilizing coordinates and angles of corresponding minutiae pairs. Orientation matching score can be computed by similarity function, as in

$$
M o=\frac{1}{N} \sum_{i \in \Pi} \sum_{j \in \Pi} e^{-\frac{d \phi\left(O(i, j)-O^{T}(i, j)\right)}{\mu}}
$$

where $O(i, j)$ denotes the direction of block $(i, j)$ in reference fingerprint image, while $O^{T}(i, j)$ is the direction of the corresponding block in the input image, function $d \phi(\alpha, \beta)$ is defined as an acute angle that locates between $\alpha$ and $\beta, \Pi$ indicates the overlapped area between two images, $N$ is the number of the overlapped blocks, $\mu$ is an 
empirical value. Therefore, the matching score based on orientation features between two fingerprint images is normalized to a range of $(0,1]$ that can be conducted fusion with minutiae matching score which is given by (1) at the confidence level.

\section{Two Stage Matching Scheme}

Now we define the strategy of two stage fingerprint recognition. At the first stage, fingerprint images are classified by using the minutiae based matching. The final decision result can be given in the first stage in most cases with fair average quality fingerprint images because of improved discriminative capability of minutiae based classifier. The output of the first stage is used as the input of next stage with reject option under the condition of $T_{L}<M_{s}<T_{H}$, in which $T_{L}$ and $T_{H}$ is set considering trade off between accuracy and efficiency of fingerprint recognition system. In practice, it is confidently believed that the input fingerprint is from genuine owner if the matching score is larger than $T_{H}$, while the subject is regarded as imposter if it is less than $T_{L}$ and there is no further action is required.

In the second stage, both the matching scores of minutiae and orientation features are utilized as inputs of higher stage classification system for that the minutiae features are more informative while the orientation matcher is complementary to minutiae matcher when it is uncertain of the final decision. Because of limited information for classifiers, theoretical model for fusion is simplified and three approaches to combing matching scores are adopted, that is:

\section{A. Score Summation}

$$
M=\frac{M s+M o}{2} .
$$

\section{B. Score Product}

$$
M=M s \square M o \text {. }
$$

\section{Fusion based on D-S Evidence Theory.}

This method of information fusion is based on D-S evidence theory which is a decision-making theory that makes decision by combining several evidences. Compared with other traditional probability theories, an important aspect of this theory is the combination of evidence obtained from multiple sources and the modeling of conflict between them. The method of combining two classifiers based on D$\mathrm{S}$ evidence theory can be briefly described as follows.

$$
M=\frac{M s \square M o}{(1-M o(1-M s)-(1-M o) M s)},
$$

where Ms and Mo are matching scores based on minutiae and orientation computed by (1) and (4) respectively.

\section{EXPERIMENTS AND CONCLUSIONS}

The algorithm discussed in this paper has been tested on the public database of fingerprint images DB3 set A in FVC2002 [10]. To compare the performance of all methods aforementioned with other alternative algorithm, ROC curves are demonstrated in Fig. 2, which in turn are cascading classifier with fusion based on score product, cascading classifier with fusion based on score summation, cascading classifier with fusion based on D-S evidence theory, minutiae triangle matching algorithm and local minutiae structure based algorithm [11].

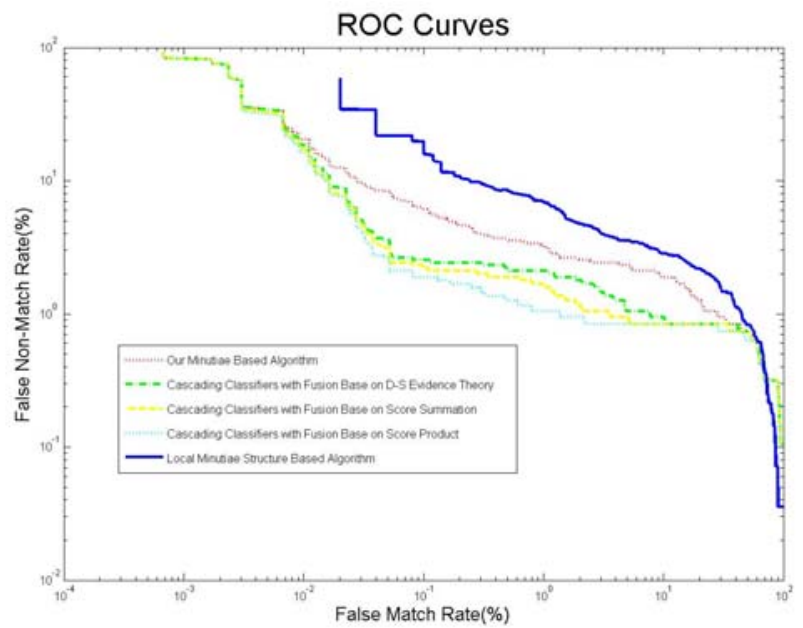

Figure 2. ROC curves of different classification systems.

As is shown in the figure, we can see that our two stage cascading schemes with information fusion at the second stage outperform all single classifiers, while cascading classifier with fusion based on score product achieves the best performance. It can also be learned that the performance of fingerprint recognition system is greatly enhanced owing to combing minutiae triangle matching with belief function of singular features .Both curves of cascading algorithm and that of single classifier are partially overlapped because that the entrance to the second stage is opened only if $T_{L}<M_{s}<T_{H}$ is fulfilled, in which $T_{L}=0.35, T_{H}=0.5$ for DB3 empirically. As far as the parameters aforesaid are concerned, our strategy is quite efficient for that the possibility of starting the second stage is less than $8 \%$ in DB3 set A statistically that is a small probability in application to fingerprint recognition system.

In Conclusion, the paper presents an algorithm for fingerprint recognition cascading minutiae and orientation based classifier in two stage. In most cases, fingerprint images can be classified confidently at the first stage by 
using the minutiae triangle matching proposed in this paper. There is a small probability in application to start the second stage in which three simplified rules of information fusion are adopted. Experimental results achieved on the public database show that all of three methods lead to a greatly improvement in the global recognition performance, while the rule of score product performs the best. However, the method of fusion based on D-S theory do not perform very well because D-S evidence theory can be employed to obtain high accuracy combining the results of two classifiers only if they have no relativity, whereas the algorithms based on minutiae and orientation do not separate absolutely in the system, that is what we should do next to improve it in this aspect.

\section{REFERENCES}

[1] Karthik Nandakumar. IEEE Proceedings of Biometrics (ICB), 5th IAPR International Conference on Biometrics Compendium, 2012, Page(s): $216-221$

[2] Jain, A.K., Yi Chen, Demirkus, M. Pores and Ridges: HighResolution Fingerprint Matching Using Level 3 Features. IEEE Transactions on Pattern Analysis and Machine Intelligence, Volume: 29, Issue: 1, Jan. 2007, Page(s): $15-27$.

[3] Jain, A.K., A. Ross and S. Prabhakar. Fingerprint matching using minutiae and texture features. Proceedings of International Conference on Image Processing (ICIP), Greece, 2001, vol. 3, pp. 282 $-285$.

[4] P. Pudil, J. Novovicova, S.Blaha and J. Kittler. Multistage Pattern Recognition with Rejection Option. Proceedings of the 11th
International Conference on Pattern Recognition, Vol.B, pp. 92 - 95, 1992.

[5] C. Kaynak and E. Alpaydin. MultiStage Cascading of Multiple Classifiers: One Man's Noise is Another Man's Data. Proc. 17th International Conf. on Machine Learning, 2000.

[6] Zhenan Sun, Yunhong Wang, Tieniu Tan and Jiali Cui. Cascading Statistical And Structural Classifiers For Iris Recognition. Proceedings of IEEE International Conference on Image Processing, 2004, pp.1261 - 1264.

[7] Jin Qi, Zhongchao shi, Xuying Zhao and Yangsheng Wang. Cascading a Couple of Registration Methods for a High Accurate Fingerprint Verification System. Proceedings of Sinobiometrics'04, LNCS 3338, Beijing, China, Dec. 2004

[8] Xuying Zhao, Xiaokun Zhang, Geng Zhao, Xiaodong Li, Kejun Zhang and Rong Qian. Triangle Matching Combined with Singular Features in Fingerprints. IEEE 2011 International Conference in Mechatronic Sciences, Electric Engineering and Computer (MEC 2011).

[9] Xuying Zhao, Xiaokun Zhang, Geng Zhao, Rong Qian, Xiaodong Li and Kejun Zhang. Fingerprint Orientation Template Matching Based on Mutual Information. Springer 2011 International Conference on Computer, Informatics, Cybernetics and Applications (CICA 2011).

[10] D. Maio, D. Maltoni, R. Cappelli, J.L. Wayman and A.K. Jain. FVC2002: Second Fingerprint Verification Competition, in proceedings of 16th International Conference on Pattern Recognition, Qubec City, vol.3, pp. 811 - 814, 2002.

[11] X. Jiang and W.Y. Yau. Fingerprint minutiae matching based on the local and global structures. IEEE 15th International Conference on Pattern Recognition, 2:1042 - 1045, September 2000 